\title{
INVARIANT SUBSPACES OF VON NEUMANN ALGEBRAS. II
}

\author{
COSTEL PELIGRAD
}

\begin{abstract}
It is shown that every parareductive operator algebra $A \subset$ $B(H)$ (as defined below) is a von Neumann algebra. For the proof of this result, some new properties of paraclosed operators are obtained. Finally, a sufficient condition that a reductive algebra be a von Neumann algebra is given.
\end{abstract}

In what follows, $H$ denotes a complex Hilbert space. The algebra of all bounded operators on $H$ is denoted by $B(H)$. If $A \subset B(H), A^{\prime}$ denotes the commutant of $A$, and $A^{\prime \prime}$ the bicommutant. Also, we denote by $M_{A}$ the von Neumann algebra generated by $A$. For every $n \in \mathbf{N}$, we denote $H^{(n)}=$ $\bigoplus_{i=1}^{n} H_{i}$ where $H_{i}=H$ for every $i, 1 \leqslant i \leqslant n$, and

$$
A^{(n)}=\{\underbrace{a \oplus a \oplus \cdots \oplus a}_{n} \mid a \in A\} .
$$

The collection of all closed linear subspaces of $H$ invariant under $A$ (i.e. invariant under every $a \in A$ ) is denoted by Lat $A$. A weakly closed algebra $A \subset B(H)$ is reductive [8] if $1 \in A$, and Lat $A=$ Lat $M_{A}$. A linear subspace $K \subset H$ is paraclosed [4] if there exist a Hilbert space $H_{0}$ and a bounded linear operator $Q: H_{0} \mapsto H$ such that $Q H_{0}=K$. The collection of all paraclosed subspaces of $H$, invariant under $A$ is denoted $\mathrm{Lat}_{1 / 2} A$. A weakly closed algebra $A \subset B(H)$ will be called parareductive if $1 \in A$, and $\mathrm{Lat}_{1 / 2} A$ $=\mathrm{Lat}_{1 / 2} M_{A}$. In this paper (Theorem 2.1) we show that if $A$ is a parareductive algebra then $A$ is a von Neumann algebra. In order to prove this result, in $\S 1$, we give some new results on paraclosed operators.

Finally, in $\$ 3$ we prove a result, announced (without proof) in [7].

Since the paper was written a proof of Theorem 2.1 in the separable case has appeared [1]. Our approach covering the possibly nonseparable case is entirely different and in fact simplifies Azoff's proof. I am indebted to the referee for calling my attention to Azoff's paper and for the suggestion that Theorem 2.1 can be formulated in this general form.

1. Paraclosed operators. Let $H_{1}, H$ be Hilbert spaces. A linear transformation $S: \mathscr{D}_{S} \mapsto H\left(\mathscr{D}_{S} \subset H_{1}\right)$ is paraclosed [4] if its graph $\Gamma_{S}=\{\xi \oplus S \xi \mid \xi \in$

Received by the editors August 8, 1977 and, in revised form, January 30, 1978 and June 15, 1978.

AMS (MOS) subject classifications (1970). Primary 46L15, 47C15; Secondary 16A32.

Key words and phrases. Reductive algebra, parareductive algebra, von Neumann algebra, paraclosed operator. 0002-9939/79/0000-0111/\$02.25 
$\left.\mathscr{D}_{S}\right\}$ is a paraclosed subspace of $H_{1} \oplus H$.

A linear transformation $S: \mathscr{D}_{S} \mapsto H\left(\mathscr{D}_{S} \subset H_{1}\right)$ is semiclosed [3] if there exist a Hilbert space $H_{2}$ and two closed operators $S_{1}: \mathscr{D}_{s} \mapsto H_{2}, S_{2}: \mathscr{D}_{S_{2}} \mapsto H\left(\mathscr{D}_{S_{2}}\right.$ $\subset \mathrm{H}_{2}$ ) such that $S=S_{2} S_{1}$. The following proposition shows that these two notions are equivalent.

1.1. Proposition. Let $H_{1}, H$ be Hilbert spaces, and let $S: \mathscr{D}_{S} \mapsto H\left(\mathscr{D}_{S} \subset\right.$ $H_{1}$ ) be a linear transformation. The following are equivalent:

(a) $S$ is paraclosed,

(b) $S$ is semiclosed.

Proof. $(\mathrm{a}) \Rightarrow(\mathrm{b})$. Since $S$ is paraclosed, its graph $\Gamma_{S}$ is a paraclosed subspace of $H_{1} \oplus H$. By the definition of paraclosed subspaces, there exist a Hilbert space $H_{2}$ and a bounded linear operator $Q: H_{2} \mapsto H_{1} \oplus H$ such that $Q H_{2}=\Gamma_{s}$. We may suppose that $Q$ is injective, since otherwise we replace $\mathrm{H}_{2}$ by $(\operatorname{ker} Q)^{\perp}$. Then, for every $\xi \in \mathscr{D}_{S}$ there exists a unique $S_{1}(\xi) \in H_{2}$ such that $Q S_{1}(\xi)=\xi \oplus S(\xi)$. Obviously, $S_{1}$ is a linear transformation $S_{1}: \mathscr{D}_{S} \mapsto H_{2}$. Moreover, $S_{1}$ is a closed operator with $\mathscr{D}_{S_{1}}=\mathscr{D}_{s}$. Indeed, let $\left\{\xi_{n}\right\}_{n=1}^{\infty} \subset \mathscr{D}_{S}$ be a sequence such that $\lim _{n} \xi_{n}=\xi_{0}$ and $\lim _{n} S_{1}\left(\xi_{n}\right)=\eta_{0}$. Since $Q$ is continuous, it follows that $\lim _{n} Q S_{1}\left(\xi_{n}\right)=Q\left(\eta_{0}\right)$. On the other hand $Q S_{1}\left(\xi_{n}\right)=\xi_{n} \oplus$ $S\left(\xi_{n}\right)$ and hence $Q\left(\eta_{0}\right)=\xi_{0} \oplus S \xi_{0}$. It follows that $\xi_{0} \in \mathscr{D}_{S}$, and $S_{1}\left(\xi_{0}\right)=\eta_{0}$. Therefore $S_{1}$ is closed and $\mathscr{D}_{S_{1}}=\mathscr{D}_{S}$. If $p_{H}$ is the projection of $H_{1} \oplus H$ onto $H$, then $S_{2}=p_{H} Q$ is a bounded operator, and $S=S_{2} S_{1}$, whence $S$ is semiclosed.

(b) $\Rightarrow$ (a). Let $H_{2}$ be a Hilbert space and let $S_{1}: \mathscr{D}_{S} \mapsto H_{2}, S_{2}: \mathscr{D}_{S_{2}} \mapsto H\left(\mathscr{D}_{S_{2}}\right.$ $\subset H_{2}$ ) be closed operators such that $S=S_{2} S_{1}$. Let $H_{0}=H_{1} \oplus H_{2} \oplus H$, and $H_{3}=\left\{\xi \oplus S_{1} \xi \oplus S \xi \mid \xi \in \mathscr{D}_{S}\right\}$. If $q$ is the projection of $H_{0}$ onto $H_{1} \oplus\{0\} \oplus$ $H$, we see that $q p_{H_{3}} H_{0}=\Gamma_{S}$ and therefore $S$ is paraclosed, which completes the proof of proposition. In [3] it is proved that if $S$ and $T$ are semiclosed operators, then so are $S+T$ and $S T$ (whenever the latter are defined). Therefore

1.2. CoROllaRY. If $S$ and $T$ are paraclosed operators, then so are $S+T$ and $S T$ (whenever the latter are defined). We need also the following:

1.3. Proposition [4]. Let $S: \mathscr{D}_{S} \mapsto H\left(\mathscr{D}_{S} \subset H_{1}\right)$ be a paraclosed operator. If $\mathscr{D}_{S}$ is closed, then $S$ is continuous.

\section{Parareductive algebras.}

2.1. Theorem. Let $A \subset B(H)$ be a parareductive algebra. Then $A=M_{A}$.

For the proof of this theorem we need some preliminary results.

Recall that a von Neumann algebra $N$ has property (P) if for every $x \in B(H)$ the weakly closed convex hull of $\left\{u^{*} x u \mid u \in N\right.$, unitary $\}$ has nonvoid intersection with the commutant $N^{\prime}$ of $N$ [9]. It is known that every discrete von Neumann algebra has property $(P)[9]$. 
The next lemma is due to D. Voiculescu [12]. We include his proof for the convenience of the reader.

2.2. LemMA. Let $Z$ be a commutative von Neumann algebra, and let $A$ be $a$ parareductive algebra such that $Z \subset A \subset Z^{\prime}$ and $M_{A}=Z^{\prime}$. Then $A^{\prime}=Z$.

Proof. Since $Z \subset A \subset Z^{\prime}$, it follows that $Z \subset A^{\prime} \subset Z^{\prime}$. So, to prove the lemma, we must show that $A^{\prime} \subset Z$. Let $t \in A^{\prime}$. Then, by [10, Proposition 6.4], there exists $z \in Z$, such that for every projection $p \in Z$, the element $(z-t) p$ has no inverse in the algebra $Z^{\prime} p$. Obviously $\operatorname{ker}(z-t)$ Lat $A \subset \operatorname{Lat}_{1 / 2} A=$ Lat $_{1 / 2} Z^{\prime}$ and therefore the projection $p_{0}$ onto $\operatorname{ker}(z-t)$ is in $Z$. The element $(z-t)\left(1-p_{0}\right)$ of $Z^{\prime}\left(1-p_{0}\right)$ is injective. We show that it is equal to zero. Indeed, if this is not true, then $0 \neq \operatorname{Range}\left[(z-t)\left(1-p_{0}\right)\right] \in \operatorname{Lat}_{1 / 2} Z^{\prime}(1-$ $\left.p_{0}\right)$. By [11, Théorème 2] it follows that there exists a positive $z_{0} \in Z\left(1-p_{0}\right)$ such that Range $\left[(z-t)\left(1-p_{0}\right)\right]=$ Range $z_{0}$. By the spectral theorem, it follows that there exists a spectral projection $0 \neq p \leqslant 1-p_{0}$ of $z_{0}$ such that

$$
p H \subset \text { Range } z_{0}=\operatorname{Range}\left[(z-t)\left(1-p_{0}\right)\right] \text {. }
$$

Then $(z-t) p$ is invertible in $Z^{\prime} p$ which is impossible. Therefore $(z-t)(1-$ $\left.p_{0}\right)=0$, and hence $t=z \in Z$.

2.3. Lemma. Let $A \subset B(H)$ be a parareductive algebra. Then $A^{\prime}=M_{A}^{\prime}$.

Proof. Since $A$ is parareductive, it follows that $A$ is reductive. Let $Z$ be the center of $M_{A}$. According to [5, Corollary 1] we have $Z \subset A^{\prime \prime}$. Since $A \subset A^{\prime \prime}$ $\subset M_{A}$ and $A$ is parareductive, $A^{\prime \prime}$ is parareductive too.

Let $p \in Z$ be the maximal projection such that $M_{A} p$ is abelian and of infinite uniform multiplicity. Then, by [5, Theorem 3] it follows that [(1$p) A]^{\prime}=(1-p) M_{A}^{\prime}$. Therefore $(1-p) A^{\prime}=(1-p) M_{A}^{\prime}$.

By the preceding lemma, we have $\left(p A^{\prime \prime}\right)^{\prime}=p M_{A}^{\prime}$, so $p A^{\prime}=p M_{A}^{\prime}$. Hence $A^{\prime}=M^{\prime}$.

The following lemma is a consequence of [7, Corollary 1.3].

2.4. Lemma. Let $A \subset B(H)$ be a parareductive algebra, and $b \in M$. Suppose that every paraclosed, densely defined operator that commutes with $A$, commutes also with $b$. Then $b \in A$.

2.5. Proposition. Let $A \subset B(H)$ be a parareductive algebra. If $M_{A}$ has property (P), then $A=M_{A}$.

Proof. We shall verify the hypothesis of Lemma 2.4. By Lemma 2.3 we have $A^{\prime}=M_{A}^{\prime}$. Let $S$ : $\mathscr{D}_{S} \mapsto H\left(\mathscr{D}_{S} \subset H\right)$ be a paraclosed densely defined operator, that commutes with $A$.

It is easy to see that $\mathscr{D}_{S} \in \mathrm{Lat}_{1 / 2} A=\mathrm{Lat}_{1 / 2} M_{A}$. By [11, Théorème 2] there exists $m^{\prime} \in M_{A}^{\prime}, m^{\prime} \geqslant 0$ such that $\mathscr{D}_{s}=m^{\prime} H$. Since $s$ is paraclosed and $m^{\prime}$ continuous, by Corollary 1.2 , it follows that $S m^{\prime}$ is a paraclosed operator. Since $\mathscr{D}_{S m^{\prime}}=H$, by Proposition 1.3 we have $S m^{\prime}$ continuous. Since $S$ commutes with $A$ and $m^{\prime} \in M_{A}^{\prime}=A^{\prime}$, it follows that $S m^{\prime} \in A^{\prime}=M_{A}^{\prime}$. 
Now, we show that $S$ commutes with $M_{A}$. Let $m \in M_{A}$. Then we have:

$$
m S m^{\prime}=S m^{\prime} m=S m m^{\prime} \text {. }
$$

Therefore $m S=S m$ on $m^{\prime} H=\mathscr{Q}_{S}$ and the proposition is proved.

2.6. Corollary. Let $A \subset B(H)$ be a parareductive algebra. If $M_{A}$ is a discrete von Neumann algebra, then $A=M_{A}$.

Proof. Since every discrete von Neumann algebra has property (P), the corollary follows immediately from Proposition 2.6.

The following lemma is a consequence of [1, Proposition 4.1].

2.7. Lemma. Let $A \subset B(H)$ be a parareductive algebra, and $Z$ the center of $M_{A}$. If $p \in Z$ is the projection such that $p M_{A} p$ is discrete and $(1-p) M_{A}(1-$ $p)$ is continuous, then $p \in A$.

The proof of Theorem 2.1 follows from Corollary 2.6., [7, Corollary 1.3] or [11, Théorème 1], and Lemma 2.7.

2.8. Corollary. Let $A \subset B(H)$ be a weakly closed algebra such that $1 \in A$. If every linear subspace of $H$ invariant under $A$ is invariant under $M_{A}$, then $A=M_{A}$.

This corollary has been obtained independently by E. Azoff [2].

3. Reductive algebras. In [7, Theorem 2.2] the following is proved.

3.1. THEOREM. Let $A \subset B(H)$ be an algebra with the following properties:

(a) $A^{(2)}$ is reductive,

(b) $A^{(2)}$ contains a von Neumann algebra $N$ with the property (P) and having finite commutant.

Then $A=M_{A}$.

At the end of the same paper, we claimed (without proof) that the following improvement of this theorem can be given (see the proof below):

3.2. TheOREM. Let $A \subset B(H)$ be an algebra with the following properties:

(a) $A^{(2)}$ is reductive,

(b) $A$ contains a von Neumann algebra $N$ having finite commutant.

Then $A=M_{A}$.

We recall that a linear transformation $T: \mathscr{D}_{T} \mapsto H,\left(\mathscr{D}_{T} \subset H\right)$ is a graph transformation for $A$ [8] if there exist $n \in \mathbf{N}$ and linear transformations $\left\{T_{i}\right\}_{i=1}^{n-2}$ defined on $\mathscr{D}_{T}$ such that $\left\{\xi \oplus T \xi \oplus T_{1} \xi \oplus \cdots \oplus T_{n-2} \xi \mid \xi \in \mathscr{D}_{T}\right\}$ $\in$ Lat $A^{(n)}$.

To prove the Theorem 3.2 we need the following:

3.3. Lemma. Let $N \subset B(H)$ be a von Neumann algebra, having finite commutant. Then, every densely defined graph transformation for $N$, is preclosed. 
Proof. Let $T$ be a densely defined graph transformation of $N$ and let $\left\{T_{i}\right\}_{i=1}^{n-2}$ be linear transformations defined on $\mathscr{D}_{T}$ such that $\left\{\xi \oplus T \xi \oplus T_{1} \xi\right.$ $\left.\oplus \cdots \oplus T_{n-2} \xi \mid \xi \in \mathscr{D}_{T}\right\} \in$ Lat $N^{(n)}$. If $\Delta_{n-1}$ is the diagonal of $H^{(n-1)}$, then it is easy to see that the transformation $\tilde{T}:\left(\mathscr{Q}_{T}^{(n-1)} \cap \Delta_{n-1}\right) \oplus \Delta_{n-1}^{\perp} \mapsto H^{(n-1)}$ defined by:

$$
\begin{aligned}
\tilde{T}(\xi \oplus \cdots \oplus \xi) & =T \xi \oplus T_{1} \xi \oplus \cdots \oplus T_{n-2} \xi \quad \text { if } \xi \in \mathscr{D}_{T}, \\
\tilde{T}\left(\xi_{1} \oplus \cdots \oplus \xi_{n-1}\right) & =0 \oplus 0 \oplus \cdots \oplus 0 \text { if } \xi_{1} \oplus \cdots \oplus \xi_{n-1} \in \Delta_{n-1}^{\perp}
\end{aligned}
$$

is a densely defined, closed operator affiliated to $N^{(n-1)^{\prime}}$. Since $N^{\prime}$ is finite, it follows that $N^{(n-1)^{\prime}}$ is a finite von Neumann algebra. Let $p$ be the projection of $H^{2(n-1)}$ onto its $n$th component. Then $p \in N^{(n-1)^{\prime}}$. Since $\tilde{T}$ is affiliated to $N^{(n-1)^{\prime}}$, then according to [6, Theorem $\mathrm{XV}$, p. 119] we obtain that $p \tilde{T}$ is preclosed, whence $T$ is preclosed.

The proof of Theorem 3.2, is similar with the proof of [7, Theorem 2.2] applying Lemma 3.3 instead of [7, Lemma 2.3].

3.4. Corollary. Let $N, M \subset B(H)$ be two type II von Neumann algebras such that $N \subset M$ and $N^{\prime}$ is finite. Then every reductive algebra $A$ with $N \subset A \subset M$ is a von Neumann algebra.

Proof. Since $M$ is a type II von Neumann algebra, $M^{\prime}$ is also a type II von Neumann algebra. Therefore, there exist $p_{1}, p_{2} \in M^{\prime}$ orthogonal projections which are equivalent and $p_{1}+p_{2}=1$. A standard argument shows that the algebras $A$ and $\left(p_{1} A p_{1}\right)^{(2)}$ are unitarily equivalent.

Since $A$ is reductive, it follows that $\left(p_{1} A p_{1}\right)^{(2)}$ is reductive. The von Neumann algebra $p_{1} N p_{1}$ has finite commutant because $p_{1} \in M^{\prime} \subset N^{\prime}$. Therefore the algebra $p_{1} A p_{1}$ satisfies the hypothesis of Theorem 3.2. By this theorem $p_{1} A p_{1}$ is a von Neumann algebra and hence $A$ is a von Neumann algebra.

\section{REFERENCES}

1. E. A. Azoff, Invariant linear manifolds and the selfadjointness of operator algebras, Amer. J. Math. 99 (1977), 121-137.

2. Kaplanski-Hilbert modules and the self-adjointness of operator algebras (preprint).

3. S. R. Caradus, Semiclosed operators, Pacific J. Math. 44 (1973), 75-81.

4. C. Foias, Invariant para-closed subspaces, Indiana Univ. Math. J. 21 (1972), 887-906.

5. T. B. Hoover, Operator algebra with reducing invariant subspaces, Pacific J. Math. 44 (1973), 173-180.

6. J. von Neumann, Collected works. III, Pergamon Press, New York, 1961.

7. C. Peligrad, Invariant subspaces of von Neumann algebras, Acta Sci. Math. (Szeged) 37 (1975), 275-279.

8. H. Radjavi and P. Rosenthal, Invariant subspaces, Springer-Verlag, New York, 1973.

9. J. T. Schwartz, $W^{*}$-algebras, Gordon and Breach, New York, 1967.

10. S. Strătilă and L. Zsidó, An algebraic reduction theory for $W^{*}$-algebras. II, Rev. Roumaine Math. Pures Appl. 18 (1973), 407-460.

11. D. Voiculescu, Sure les sous-espaces parafermés invariants d'une algèbre de von Neumann, Bull. Sci. Math. (2) 96 (1972), 161-168.

12. , Diploma work, Univ. of Bucharest, 1972. (Romanian)

Institutul National pentru Creatie, Stinntifica si Technica, Bucuresti, Romania 